\title{
A framework for effective collaborative working
}

in construction

Q1 M. A. Shelbourn, D. Bouchlaghem, C. Anumba and P. Carrillo

The potential benefits of collaborative working in construction projects have yet to be fully realised in the sector. This is in part due to the misunderstanding of the use of the information and communication technologies (ICT) and tools associated with working collaboratively. There are, however, isolated pockets of experts that have realised that in order to fully embrace the potential of collaborative working in their businesses, the role of ICT has to be closely aligned with the people that use it, and should relate to the overall goals of the business. The aim of the Planning and Implementation of Effective Collaborative Working in the Construction (PIECC) project is to develop a decision-making framework that enables organisations to fully integrate ICT and its associated people and business issues into their projects, and individual businesses. This paper reports on the development processes used to develop the framework, and its evaluation and testing within the UK construction sector. The paper presents the different processes that should be followed to develop a joint collaboration strategy, and the results and implications of using the PIECC framework to manage collaborative working.

\section{INTRODUCTION}

The role of collaborative working in the construction sector is beginning to influence the ways of working in some organisations, and is even changing the working practices of the industry as a whole.

Collaborative working is not achievable without the use of information and communication technologies (ICT). These technologies are often simple tools available on most personal computers, such as spreadsheets and word processing, but more sophisticated tools that technological suppliers call 'project extranets' are also available. There are a number of commercial tools available on the market. There are pros and cons associated with these and it is for people to make their own choice of preference, but a good guide is provided by the Network for Construction Collaboration Technology Providers. ${ }^{1}$ This report shows the benefits of using ICT and collaborative working tools by showing that collaborative working pays.

As with previous initiatives that involve the implementation of such collaboration tools and ICTs in projects and businesses, there has been a traditional focus on the technological aspects ${ }^{2-4}$ with limited regard for the effects that these implementations have on the people using them and the organisations they work for. ${ }^{5-8}$ Implementing collaborative working in organisations cannot be achieved without the use of ICT, and conversely those implementations that concentrate purely on the socio-economic aspects (the people and business perspectives) will similarly fail. ${ }^{9-14}$

The Planning and Implementation of Effective Collaboration in Construction (PIECC) project has recognised that there needs to be a balanced approach to implementing collaborative working in projects in order to take advantage of the benefits that ICT can provide.

The PIECC project was a 2 year research and development project that involved Loughborough University and a number of leading construction organisations-ARUP; Buro Happold; Capita Symonds; Mott MacDonald; Taylor Woodrow; and the AVANTI Programme. Through the extensive involvement of the industrial partners a framework was developed that enabled managers to plan and implement collaborative working into their projects. A copy of the framework is available from http://piecc.lboro.ac.uk/ Results/PIECC\%20Brochure.pdf or by contacting any of the authors.

Socio-technical aspects of ICT implementations also need to be given full consideration. The project has a focus on supporting strategic decision-making by highlighting areas where collaborative working can be improved and incorporating the organisational (business), project and users needs. The needs of the construction industry have been considered as a basis for developing a framework that facilitates the strategic planning and implementation of effective collaborative working policies and protocols. ${ }^{15}$ When carefully planned and, if based on informed decisions, it is believed that these policies and protocols will help organisations improve their collaborative working activities, achieve better benefits from it, and maximise the use of tools and techniques that are currently available commercially. The remainder of this paper describes how the balanced approach, shown in Fig. 1, is achieved through the development of a collaboration strategy.

\section{THE PIECC PROJECT}

The construction industry was initially slow in the uptake of ICT to improve the performance of its projects; however, during the years 


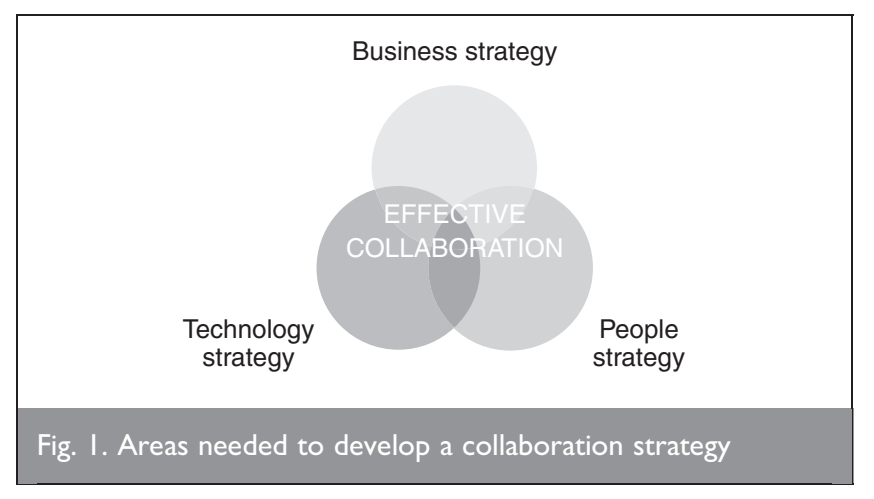

2000-2005 there has been a marked increase in its use. ${ }^{16}$ The introduction of ICT has enabled the phenomenon of collaborative working to become part of the industry's every-day practices. With the need to demonstrate the complexities of introducing collaborative working into projects proving difficult to establish, the PIECC project aims to show how a more strategic approach to using ICT systems for collaboration on construction projects is achievable.

\section{PIECC PROJECT RESEARCH METHODOLOGY}

In order to complete the objectives of the PIECC project a combination of recognised research methods were employed on the project. These included the following activities.

(a) Use of published resources-through an extensive literature review the current 'state-of-the-art' in collaborative working was determined, ${ }^{17}$ which included the change in management aspects ${ }^{18}$ and the technologies used. ${ }^{19}$

(b) Field studies-with key experts in collaborative working from the industry, and the accumulation of case material from existing projects. These led to the identification of the requirements for effective collaborative working and the key issues to address from the industry perspective. ${ }^{15}$

(c) Use of a 'develop-test-refine' strategy (action research) to improve the framework to be introduced into construction projects. How this was achieved is the main focus of this paper.

\section{DEVELOPMENT OF THE PIECC FRAMEWORK}

Shelbourn et al. ${ }^{15}$ documents the needs and requirements of the industry for effective collaboration. Briefly, these were categorised into six groups: model; process; standards; good practice; design; and legal aspects. The needs and requirements were drawn from information gained from a questionnaire, and semi-structured interviews with leading experts. ${ }^{8}$

With the requirements gained the research team moved their efforts into developing a decision-making framework for effective collaborative working. Intense discussions with the industrial partners, via the project steering group, and other prominent members of the construction industry revealed that a 'developtest-refine' approach was the most appropriate for this stage of the research.

From the literature review results ${ }^{17-19}$ and the user requirements report ${ }^{15}$ initial discussions revealed a strong idea that there are essentially four key stages to follow. These are shown in Fig. 2.

The first iteration built upon this initial idea with the research team integrating the six key factors (determined in the PIECC literature review) for effective collaboration: vision; engagement;

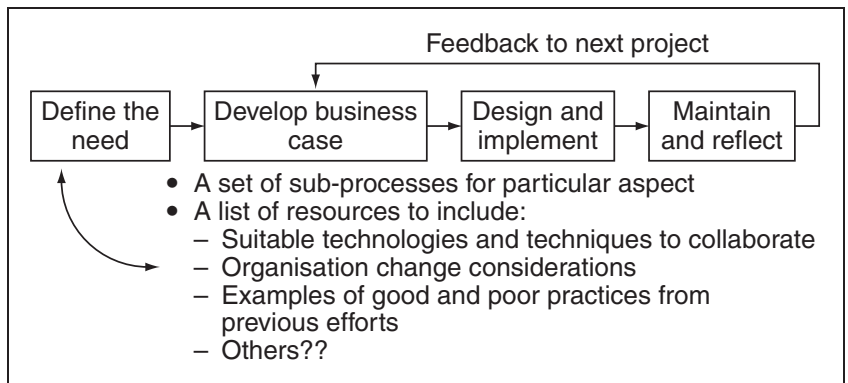

Fig. 2. Initial thoughts of the decision-making framework

trust; communication; processes; and technologies-into the four key areas.

Comments and feedback from the industrial partners on the initial versions of the framework were encouraging. They commented that they were encouraged by the development, but questioned whether the framework would not be better served having a more process-centric focus. This question was addressed by the research team in the second iteration of framework development. The industrial partners were complementary in their assessments of using additional information to aid the process of effective collaborative working.

The second cycle began with the redesign of the framework to show greater process focus to the framework. The framework was split into three distinct stages. The first aimed to bring together the different organisations involved in the collaboration to 'align their business strategies'. The second stage requires the collaboration champion or team to define the three strategies for effective collaboration (i.e. 'people', 'project' and 'technology' strategies). Once these have been defined the collaboration begins and is continuously monitored to make sure that all participants are working in the agreed ways. Once the collaboration has ended, feedback is given to the business on how to improve current practices.

The end of iteration two of the framework development cycle showed that the additional information-how it was presented, and what information was included-is of vital importance to the success of the framework. The project steering group had some reservations on the content of the supporting information, in that it had been written in an 'academic' style. It was agreed that each project partner should take ownership of one stage of the framework and suggest ways of making the pages more 'industry friendly'. This process enabled the supporting information to become a better supportive tool to the framework.

The industry steering group commented that version three adequately represented the key processes and supporting information to enable effective collaborative working. Despite this, members of the group had difficulties with understanding how the framework could be used, and where key decisions needed to be made.

During a key project progress meeting the research team and industrial partners created a 'rich picture' of how the processes must be linked together in a more usable format. After further discussions the 'rich picture' was transformed into the framework shown in Fig. 3. This version is the final version of the PIECC decision-making framework. 


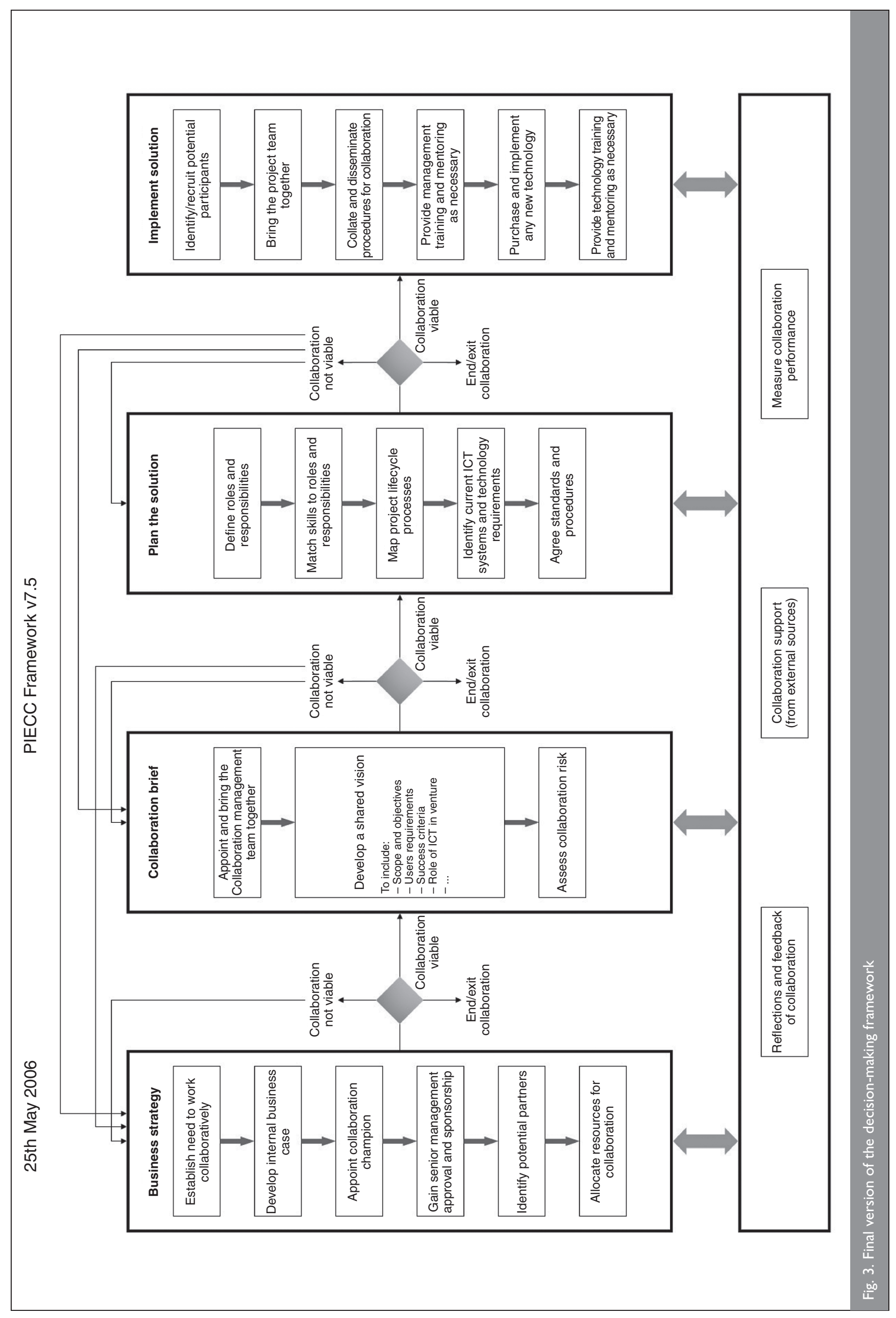


The framework has four distinct sections in which collaborating organisations set up their 'business strategy'; 'define a collaboration brief'; 'plan a solution' to fulfil that brief; and then 'implement that solution'. The collaborators do this by working from the top of each section through the described processes to develop procedures of increasing detail (as they move from the left to the right) for their collaboration. Before progressing to the next phase of the collaboration, a decision is needed to determine whether it is feasible to continue or not, dependent on the outcomes of completing the different processes in that section of the framework. If 'no' then the members of the collaboration revisit that section to try and remove the barriers or problem areas as to why the collaboration cannot proceed. If it is perceived that the collaboration can continue then the collaborators move into the next section of the framework to complete their procedures.

\section{PIECC FRAMEWORK EVALUATION AND VALIDATION}

Figure 4 shows a graphical representation of the evaluation method. This method was chosen to first evaluate the significance of the framework developed through careful appraisal and study; and secondly to validate the evaluation process to show improvements in the planning and implementation of collaborative working in construction projects.

The first approach concentrated on industrial experts evaluating and commenting on the potential use of the framework within their organisations and projects. The second approach concentrated on inviting key industrial representatives to an all-day workshop. The aim of the workshop was to identify and clarify the issues surrounding the impacts of ineffective collaboration on project performance, and how the PIECC framework could address these issues. The third approach concentrated on introducing the PIECC framework to real construction projects to assess the impacts it may have.

The next sections describe the results from each of these stages.

\section{I. Semi-structured interview results}

The project team returned to a number of the key personnel originally interviewed in the project to gain their feedback on the development of the PIECC framework.

The interviews highlighted the need for four clear ingredients to achieve effective collaborative working. These were

(a) changing the culture (of the industry)

(b) introducing enabling technologies (c) promoting new contractual relationships

(d) managing the risks (of collaboration).

Discussions in the interviews highlighted those aspects of the PIECC framework that stood out as enabling effective planning and implementation of collaborative working. The following sections describe which of the twenty-three processes the interviewees particularly felt were important to successful planning and implementation of effective collaborative working into projects.

5.1.1. General issues. The interviewees recognised the need to begin using the PIECC framework as early as possible in the project life-cycle; preferably it should be used as a tool to bring the consortium together to develop bids for winning projects. It has been the experience of one interviewee that 'all too often the project is too far down the line to warrant using the framework.... For the framework to succeed the interviewee felt that there needs to be a '... change in the culture (of the industry) from just jumping in and doing it...' to a more '... strategic approach to (collaborative) working....

This strategic approach will inevitably mean a significant change in managing the project output to include the new ways of working associated with collaborative working.

The interviewees agreed that the definition, and bringing together of, a coherent 'business strategy' was an important ingredient to the success of collaborative working. There were a number of areas that the interviewees felt where the PIECC framework can provide significant improvements.

Firstly, the question of 'what does it mean to me?' has to be clearly defined in the processes and procedures of the collaboration strategy. The individuals involved in the collaboration need to have an input into how the collaboration 
is to work on a day-to-day basis, and to understand how it will affect them in their daily working activities.

This issue also affects another clearly important consideration in the success of collaborative working-what are the 'ramifications of not disseminating the business strategy to the rest of the organisation?' The PIECC framework stresses the importance of disseminating the following information.

(a) How the collaborative working venture will affect the organisation, and its associated groups, goals and objectives.

(b) That the business, people, and ICT strategies for all participating organisations need to be aligned for collaboration to be effective in the project.

One interviewee felt that leadership '.. is the core issue to successful project collaborations'. The ability to delegate responsibility to others in the collaborative working venture is an essential quality of any manager in this role. The leader of the collaborative working venture-PIECC calls them a 'collaboration champion'-must understand the importance of building trust within the team. The interviewee went on to stress that (in their opinion) leaders that are good, '... tend to be born not raised...', which leaves the industry with a difficult question to answer: 'how do you develop the people to set the collaboration up and implement the solution?'-a question that is beyond the scope of the current research.

The following list shows those of the specific processes of the PIECC framework that were deemed essential by the interviewees.

(a) Appointing a champion is seen as essential to the successful planning and implementation of effective collaborative working in projects. One interviewee stressed that this person needs to be an enthusiastic, strong-minded person who must be highly motivated and have excellent leadership skills. The interviewee described such a person as being 'few-and-far between' in the current climate of the construction industry.

(b) Developing a shared vision to enable success, all stakeholders in the project must agree a vision for the project. This vision must include descriptions as to what happens when the collaboration goes wrong. The vision must include information that has a 'common' meaning and understanding for all.

(c) Defining roles and responsibilities-it is vitally important to know that required resources are in place, and made available, to enable the management team of the collaboration to recruit the right people to work together. Once these people have been recruited their roles and responsibilities need to be fully understood by themselves, and all other stakeholders;

(d) Measuring collaboration performance-one interviewee highlighted the need for a subjective view of the team. This means that the performance of the collaborative working project can be measured and (potentially) shown to be effective for this type of working. The need for close monitoring of the financial arrangements of the collaborative working venture was also highlighted as an important aspect to the success of the collaboration.

5.1.2. Interviews summary. The interviewees were very appreciative of the efforts that the research team had made to improve the planning and implementation of collaborative working on projects. Apart from the specifics described above, the interview findings can be summarised as '... the PIECC framework allows any number of initiatives to be used at the correct time, to enable effective planning and implementation of collaborative working into projects'.

\subsection{PIECC industrial workshop results}

The second part of the evaluation and validation exercise involved the organisation of a 1 day workshop where industry representatives were invited to discuss the PIECC findings. The research team used their personal contacts and those of industrial partners to invite 77 people from industry. This group of people was made up from those involved in the initial questionnaire and interview processes, as well as other industrial experts who had not previously been involved in the PIECC project. All participants had had significant experience in collaborative working in the construction sector. Of these 77, 15 agreed to attend. The participants represented the construction sector-architects, design managers, project managers; the technology sectorextranet providers; and those that have both construction and technology experience.

\section{The day was essentially split into three distinct tasks.}

(a) Task 1: participants had to indicate the primary focus of their roles in the industry-completed to enable groups to be made up for the next two tasks.

(b) Task 2: in the groups, analyse the impact of ineffective collaborative working on project performance from the Business, Project, or Technology perspective.

(c) Task 3: in the groups, assess the potential benefits of using the PIECC framework to improve project performance.

For the second task the groups were given $1 \mathrm{~h}$ to discuss the impact of ineffective collaborative working on project performance, and list their top five issues. The groups were chaired by a PIECC project industrial representative, with the results recorded by a member of the research team. With these impacts documented, the groups were then asked to highlight areas of the PIECC framework that could be used to address these impacts-Task 3 . The results from the two tasks are described in the next sections.

\subsubsection{Task 2 results. As described above, there were three} groups each addressing the impact of ineffective collaborative working on project performance from the business, project or technology perspectives. A summary of the results found is shown in Table 1.

From the table it can be seen that there are commonalities in the issues found in the business and project results. For example, the most important issue highlighted centres around building trust in the project team, and where this is found to be ineffective, there is a clear breakdown of relationships in the team. The project can also suffer from 'cost and time inefficiencies due to wasted effort and repetition of work', which in turn leads to the team suffering from a 'lack of focus' towards the project outputs. Associated with this, many projects have 'too much waste', which leads to a 'loss of value and quality' from the team in the project. Other issues shown from the results of Task 2 include a lack of 'engagement' and 'ownership' in the project team leading to poor morale and motivation. This can lead to a lack of knowledge sharing and innovation resulting in limited creativity within the project team. 


\begin{tabular}{lll} 
Business & Project & Technology \\
\hline $\begin{array}{ll}\text { Poor morale and motivation } \\
\text { Lack of focus }\end{array}$ & Breakdown of relationships & Poor communication \\
$\begin{array}{l}\text { Too much waste } \\
\text { Barriers to creativity }\end{array}$ & effort/repetition & Lack of collaborative contracts and cultures \\
Managing risk and creating trust & Loss of value/quality & Lack of standards \\
& Lack of knowledge sharing and innovation & Working in 3D-nD differences in software \\
& & functions of vendors
\end{tabular}

Table I. Summary of the results from Task 2 of the workshop

The top technological issues that cause ineffective collaborative working on projects centred around 'poor communication' from a 'lack of standards' and 'collaborative cultures and contracts'. The barriers associated with the technology issues were seen by the workshop participants to be much easier to break down than the project and business aspects.

Having determined the negative impacts that ineffective collaborative working has on project performance, Task 3 determined which of the 23 processes could be used to address the issues resulting from Task 2.

5.2.2. Task 3 results. The third task of the day was to '... assess the potential benefits of using the PIECC framework ...' to address the inefficiencies in collaborative working identified in Task 2. The participants remained in the same groups and again were given $1 \mathrm{~h}$ to discuss the key issues. After this, a rapporteur from each group described their findings to the rest of the workshop. Collation of the information gathered is shown in the next sections.

5.2.3. The business perspective. From a business perspective, the 'develop a shared vision' and 'measure collaboration performance' activities can be used to provide a reward and recognition policy to solve the poor motivation and morale aspects. The lack of focus can be addressed by the development of a shared business strategy in the early stages of the collaborative working venture that includes the development of a shared vision and assessing the risks of the venture. Barriers to creativity can be addressed by carefully managing the fluidity of the project team by bringing the right people together in the first instance, and also defining their roles and responsibilities at the outset of the venture.

\subsubsection{The project perspective. The results from a project} perspective are listed here.

(a) Cost and time inefficiencies and wasted effort are reduced by identifying partners that can work well together, providing them with the tools to set off in the same direction together by focusing on processes, procedures and core competencies that enable an efficient way of doing things to be developed.

Clarity of purpose is developed to enable efficient collaborative working within the team.

(b) Value and quality reductions-the PIECC framework allows team goals to be tied to the client values through the development of a collaboration brief. This means that the client's values are built into the processes and procedures to be adopted in the collaborative working venture. It is important to stress the importance of these values in the briefıngs and inductions of project team members. (c) Lack of ownership/poor morale/and loss of engagement can be addressed early using the PIECC framework. The bringing together of the business strategies of all organisations involved in the collaborative working venture and deciding upon a 'champion' to lead the collaboration is perhaps the most important step in the process. Other aspects that need to be addressed include: establishing the right culture for the project and, in developing the shared vision, remembering that it should be positive and set the behavioural rules (modus operandi) of the project. Ownership should be built into the roles and responsibilities, and the personal development plans of the people involved in the collaboration.

(d) Lack of knowledge sharing and innovation aspects are addressed by selecting organisations to work with that have a proven track record in this area. The collaboration strategy allows the development of roles and targets that define agreements on incentive schemes, and knowledge capture and sharing processes. These roles and targets should be highlighted to the team when they are brought together as part of training sessions for the collaborative working venture.

The technological aspects are addressed by the PIECC framework in less detail than the people and business aspects. The developments of the PIECC framework allow 'poor communications' to be removed. The development of a 'collaborative culture' is achieved by sharing goals, objectives, resources, etc. in the collaborative working venture. More detailed issues surrounding the development of standards, the software functionalities of the ICT providers, and whether to work in $3 \mathrm{D}-n \mathrm{D}$ are all outside the scope of the PIECC framework. The PIECC work acknowledges initiatives that are addressing these ICT aspects and are included in the detailed information that supports the framework.

5.2.5. Summary of evaluation and validation. To provide an overall summary of the evaluation and validation exercise for the PIECC framework it would be prudent to bring together the interviews, workshop and project results. Fig. 5 shows which of the PIECC framework's processes are the most important to effective collaborative working in projects.

The most important processes in the PIECC framework are: 'appoint a collaboration champion', 'develop a shared vision', 'define roles and responsibilities' and 'agree standards and procedures'. It should be stressed that all the processes of the PIECC framework are considered important to the success of collaborative working. 


\begin{tabular}{|c|c|}
\hline 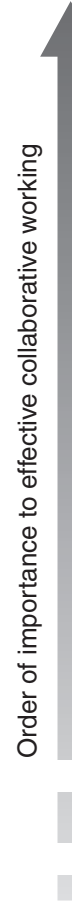 & $\begin{array}{l}\text { - Appoint a collaboration champion } \\
\text { - Develop a shared vision } \\
\text { - Define roles and responsibilities } \\
\text { - Agree standards and procedures } \\
\text { - Identify current ICT systems and technology requirements } \\
\text { - Bring the project team together } \\
\text { - Provide management training and mentoring as necessary } \\
\text { - Provide technology training and mentoring as necessary } \\
\text { - Identify potential partners } \\
\text { - Establish need to work collaboratively } \\
\text { - Assess collaboration risks } \\
\text { - Develop internal business case } \\
\text { - Gain senior management approval and sponsorship } \\
\text { - Match skills to roles and responsibilities } \\
\text { - Collate and disseminate procedures for collaboration } \\
\text { - Purchase and implement any new technology } \\
\text { - Measure collaboration performance } \\
\text { - Appoint and bring the management team together } \\
\text { - Identify/recruit potential participants } \\
\text { - Collaboration support (from external sources) } \\
\text { - Establish need to work collaboratively } \\
\text { - Map project lifecycle processes } \\
\text { - Reflections and feedback of collaboration }\end{array}$ \\
\hline & $\begin{array}{l}\text { nmary of the order of importance of the PIECC } \\
\text { for effective collaborative working }\end{array}$ \\
\hline
\end{tabular}

From the concluding discussions of the industrial workshop it was seen that the PIECC project could improve team performance, thus enabling effective collaborative working. Using Bruce Tuckman's model for building successful teams ${ }^{20}$ (Fig. 6) it was felt that if the PIECC framework was used it would probably reduce the distance between the teams' 'enthusiasm' and 'performance' during the 'forming' and 'storming' phases of the teams development. Tuckman's model would look significantly different as a result (Fig. 7).

\subsection{Perceived benefits of the PIECC framework}

From the evaluation and validation exercise the list below summarises some of the perceived benefits of the PIECC framework.

(a) PIECC is a '... good framework to provide people with the tools to develop a strategy for collaboration....

(b) PIECC '... really makes you think about collaboration....'

(c) PIECC highlights '... the inter-relationships needed to ensure a route is followed to plan and implement collaborative working....

(d) PIECC is a '... sign and marker for an organisation that will need (PIECC) to win work and carry out that work collaboratively....

(e) PIECC prevents '... people giving lip service to the uptake of collaboration, only for them to back to normal/original working practices....

As the PIECC framework is used more and more in the construction industry it is expected that the list of benefits will get longer.

\section{FURTHER WORK, RECOMMENDATIONS FOR IMPROVEMENTS}

The results from the evaluation and validation exercise have shown a positive response from the industry as to the usefulness of the PIECC framework for effective collaborative working in construction. There were, however, a number of suggested improvements and considerations.

(a) Stress the importance of why the people and process aspects are most important.

(b) Identify how to develop the people to set the collaboration up and implement the chosen solution.

(c) Evaluate and validate the framework in real construction projects/document case studies.

(d) Can the software vendors push PIECC-or is it more of the clients responsibility to do so?

(e) Integration with wider project environment/overlap with the project process/stage-RIBA Plan of Work, Process Protocol, etc.

(f) Include a health check for measuring against effective collaborative working.

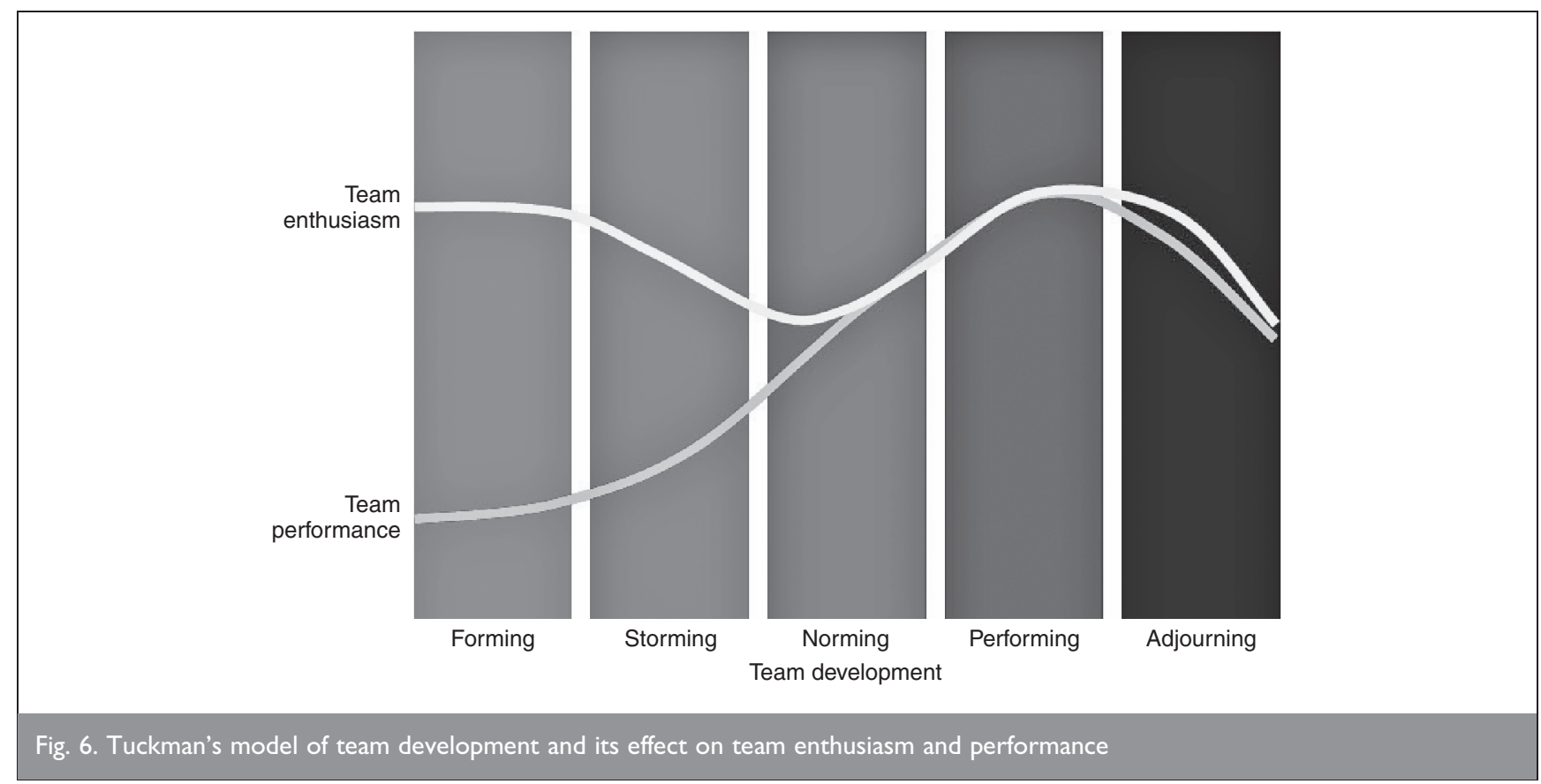




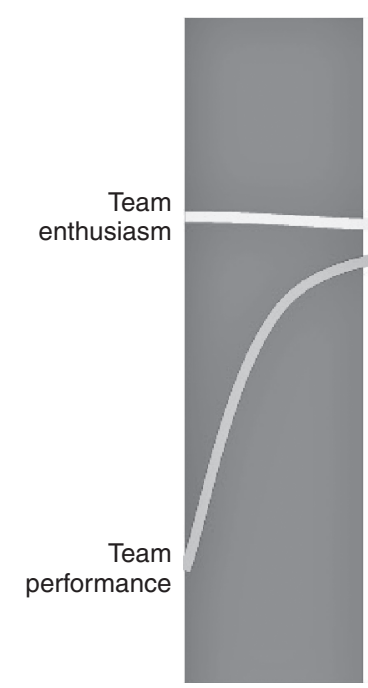

Forming

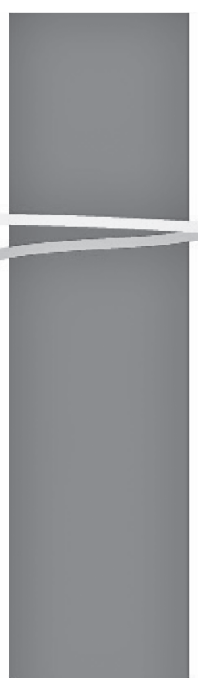

Storming

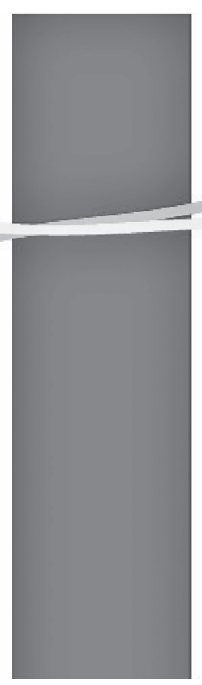

Norming

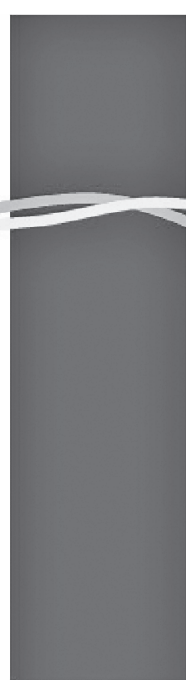

Performing

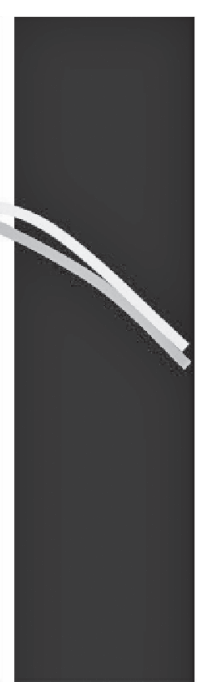

Adjourning

Team development

Fig. 7. Tuckman's model of team development and its effect on team enthusiasm and performance if PIECC is used

These suggestions and considerations will be taken up in any future developments of the PIECC framework.

\section{CONCLUSIONS}

This paper has described the methodology for the development, evaluation and validation of the PIECC decision-making framework. It has shown the iterative cycles of developing the framework through its different versions, and how these developments were commented on and influenced by the industry steering group.

Once the framework had been finalised the model used for the evaluation and validation exercise was discussed, and the results from each aspect described. The evaluation revealed a favourable industry response to the framework, with most participants in the evaluation workshop holding the view that the PIECC framework is a useful tool that brings together some new and existing methods to better plan for the implementation of effective collaboration in construction projects.

There are four main aspects of the framework that were considered essential for the success of any collaborative working venture. These are to: 'appoint a collaboration champion', 'develop a shared vision', 'define (people's) roles and responsibilities', and 'agree standards and procedures'.

Alongside these four aspects, it is essential to '... get the right people to work as part of the collaborative working venture.... People and their relationships in the collaborative working venture are crucial to a successful collaborative working project.

\section{REFERENCES}

1. Network for Construction Collaboration Technology PRoviders (NCCTP). NCCTP Proving Collaboration Pays: Study Report. 2006. Available from: http:// ncctp.constructingexcellence.org.uk/downloads/ making_collaboration_pay.pdf Accessed 17/10/2007.

2. Kvan T. Collaborative design: what is it? Automation in Construction, 2000, 9, No. 4, 409-415.
3. Woo S., LeE E. and SASADA T. The Multiuser workspace as the medium for communications in collaborative design. Automation in Construction, 2001, 10, No. 3, 303-308.

4. Faniran 0., Love P. E., Treloar G. and Anumba C. J. Methodological issues in design-construction integration. Logistics Information Management, 2001, 14, No. 5/6, 421-426.

5. Alvarez R. It was a great system: Face work and discursive construction of technology during information systems development. Information, Technology \& People, 2001, 14, No. 4, 385-405.

6. VAKola M. and WiLSON I. E. The challenge of virtual organisation: Critical success factors in dealing with constant change. Proceedings of the European Conference on Information and Communication Technology Advances and Innovation in the Knowledge Society (eSM@RT 2002 in Collaboration with CISEMIC 2002), Salford, UK (REZGUI Y., Ingirige B. and Aouad G. (eds)), 2002, Part B, pp. 264275.

7. Ferneley E., Lima C., Fies B., Rezgui Y. and Wetherill M. Inter-organisational semantic webs to enable knowledge discovery and dissemination: technical support for the social process. Proceedings of the 10th ISPE International Conference on Concurrent Engineering, Madeira, Spain, 2003, pp. 779-785.

8. Shelbourn M., Bouchlaghem D., Anumba C. J. and Carrillo P. M. Planning and implementation of effective collaboration in construction projects. Construction Innovation, 2007, 7, No. 4, 357-376.

9. GRUDIN J. Computer-supported cooperative work: its history and participation. IEEE Computer, 1994, 27, No. 5, 19-26.

10. Koschmann T., Kelson A. C., Feltovich P. J. and Barrows H. S. Computer-supported problem based learning: a principled approach to the use of computers in collaborative learning. In CSCL: Theory and Practice (Koschmann T. (ed.)). Lawrence Erlbaum, Mahwah, NJ, 1996, pp. 83-124.

11. LOOSEMORE M. Organisational behaviour during a construction crisis. International Journal of Project Management, 1998, 16, No. 2, 115-121. 
12. Winograd T. A language/action approach on the design of cooperative work. Human Computer Interaction, 1998, 3, No. 1, 3-30.

13. Eseryel D., Ganesan R. and Edmonds G. Review of computer-supported collaborative work systems. Educational Technology and Society, 2002, 5, No. 2.

14. BALDWIN A. Overcoming the barriers to the successful introduction of collaborative technologies in construction. Proceedings of the INCITE 2004 Conference Designing, Managing and Supporting Construction Projects through Innovation and IT Solutions. Langkawi, Malaysia, 2004, pp. 319-326.

15. Shelbourn M., Bouchlaghem D., Anumba C. J. and Carrillo P. M. D2-PIECC Requirements Capture ReportMethodology, and Needs for the PIECC Project Report, 2005. Available from http://piecc.lboro.ac.uk/Publications.htm Accessed 20.11.2006.

16. ADAIR K. Meeting points, Information Age-Business Briefing Magazine, www.infoconomy.com, 53, 2005, Accessed July 2006.
17. Shelbourn M., Bouchlaghem D., Anumba C. J. and Carrillo P. M. D1-PIECC Analysis and Synthesis of Literature Report, 2005. Available from http://piecc.lboro.ac.uk/ Publications.htm Accessed 20.11.2006.

18. Erdogan B., Anumba C. J., Bouchlaghem N. M. and Nielsen Y. Change management in construction. Proceedings of the Current Context, Association of Researchers in Construction Management (ARCOM), (KHosRaWSHAHI F. (ed.)), London, 2005, vol. 2, pp. 1085-1095.

19. Koseoglu 0. O., Erdogan B., Nielsen Y., Anumba C. J. and Bouchlaghem N. M. Visual information transfer using mobile IT solutions. Proceedings of the Tenth International Conference on Civil, Structural and Environmental Engineering Computing, Stirling, Scotland, 2005, vol. 1, pp. 29-30.

20. Sмiтн M. K. and Tuckman B. W. Forming, Storming, Norming and Performing in Groups, the Encyclopaedia of Informal Education, 2005, www.infed.org/thinkers/ tuckman.htm Accessed 20.11.2006.

\section{What do you think?}

To comment on this paper, please email up to 500 words to the editor at journals@ice.org.uk

Proceedings journals rely entirely on contributions sent in by civil engineers and related professionals, academics and students. Papers should be 2000-5000 words long, with adequate illustrations and references. Please visit www.thomastelford.com/journals for author guidelines and further details. 